\title{
Knockdown of SALL4 expression using RNA interference induces cell cycle arrest, enhances early apoptosis, inhibits invasion and increases chemosensitivity to temozolomide in U251 glioma cells
}

\author{
LEI ZHANG* , YONG YAN*, YING JIANG, JUN QIAN, LEI JIANG, GUOHAN HU, YICHENG LU and CHUN LUO \\ Department of Neurosurgery, Changzheng Hospital, Second Military Medical University, Shanghai 200003, P.R. China
}

Received December 3, 2015; Accepted May 11, 2017

DOI: $10.3892 / \mathrm{ol} .2017 .6722$

\begin{abstract}
Spalt-like transcription factor 4 (SALL4) is essential for the maintenance of the self-renewal and pluripotent properties in embryonic stem cells. Although the detailed mechanism remains unclear, dysregulation of SALL4 has been detected in various malignancies. Previously, the authors' of the present study reported that the expression level of SALL4 was associated with the poor prognosis of glioblastoma multiforme (GBM). The present study aimed to investigate the function of SALL4 in U251 human glioblastoma cells, including apoptosis and invasion inhibition. It was revealed that knockdown of SALL4 expression through RNA interference induced cell cycle arrest, enhanced early apoptosis and significantly inhibited invasion. Furthermore, downregulation of SALL4 was associated with a significantly lower expression level of the core transcription factors, including POU class 5 homeobox 1, SRY-box 2 and Nanog homeobox. In addition, inhibition of SALL4 significantly reduced the concentration of chemotherapeutic agent temozolomide required to inhibit cell growth by $50 \%$, which decreased from $113.66 \pm 23.07$ and $114.93 \pm 20.91 \mu \mathrm{g} / \mathrm{ml}$ to $68.34 \pm 3.52$ and $67.44 \pm 4.71 \mu \mathrm{g} / \mathrm{ml}$ in two independent short interfering RNA transfected groups. These results indicate that SALL4 serves an important role in the GBM pathophysiology and targeting SALL4 may be a potential approach to the treatment of GBM.
\end{abstract}

\section{Introduction}

Glioma is the most common subgroup type of brain tumor, with an incidence of 5-6/100,000 cases/year in the United

Correspondence to: Professor Yicheng Lu or Professor Chun Luo, Department of Neurosurgery, Changzheng Hospital, Second Military Medical University, 415 Fengyang Road, Shanghai 200003, P.R. China

E-mail: doctoradvisor@163.com

E-mail: doctorluochun@163.com

*Contributed equally

Key words: glioblastoma, U251, spalt-like transcription factor 4, short interfering RNA
States (1). Among all types of glioma, glioblastoma multiforme (GBM) accounts for $\sim 50 \%$ of cases and has the most malignant phenotype (2). Despite the extensive developments that have been invested in surgical techniques and therapeutic agents, $88 \%$ of patients with GBM succumb to this disease within 3 years (3). GBM remains one of the most challenging malignancies worldwide.

Embryonic stem cells (ESCs) are known for their potent pluripotency and are able to differentiate into $>220$ cell types in the adult body. The human homologue of the drosophila spalt-like transcription factor 4 (SALL4) is a zinc-finger transcription factor, which is responsible for maintaining pluripotency and longevity of ESCs (4-6). Previously, cancer stem cells (CSCs) have been identified in various types of malignancies $(7,8)$ and demonstrate high self-renewal capabilities able to sustain tumor growth (9). Thus far, ESCs and CSCs have been revealed to share numerous biological similarities with the SALL4 expression pattern being one of them. In various types of tumors, high SALL4 expression level has been associated with increased malignancy, including increased metastasis, enhanced proliferation (10-12) and poor differentiation $(13,14)$.

In the authors' previous study, it was demonstrated that SALL4 was highly expressed in glioma and significantly associated with poor survival (15). The present study further investigated the biological role of SALL4 in the tumorigenesis of glioma and explored the underlying mechanism of action. It was revealed that SALL4 regulated the cell cycle, apoptosis, tumor cell invasion and temozolomide (TMZ) treatment response in the U251 GBM cell line. Furthermore, decreased SALL4 expression level was associated with a decreased expression level of core transcription factors, including POU class 5 homeobox 1 (OCT4), SRY-box 2 (SOX-2) and Nanog homeobox (NANOG). Additionally, knockdown of SALL4 inhibited O-6-methylguanine-DNA methyltransferase (MGMT) and adenosine triphosphate-binding cassette subfamily G member 2 (ABCG2) expression levels, which may serve essential roles in GBM chemoresistance. These results suggest that SALL4 serves an important role in tumorigenesis and may be a useful therapeutic target for GBM.

\section{Materials and methods}

Cell culture, construction and transfection. The human malignant glioma cell line U251 was purchased from the 
Type Culture Collection of the Chinese Academy of Science (Shanghai, China). Cells were cultured in Dulbecco's modified Eagle's medium (DMEM; Invitrogen; Thermo Fisher Scientific, Inc., Waltham, MA, USA) containing $8 \%$ fetal bovine serum (Gibco; Thermo Fisher Scientific, Inc.) and antibiotics (100 U/ml penicillin $\mathrm{G}$ and $100 \mu \mathrm{g} / \mathrm{ml}$ streptomycin). A total of four different short interfering RNA (siRNA) sequences against SALL4 (GeneBank accession no. NM-020436) and one scrambled siRNA were purchased from Shanghai GenePharma Co. Ltd. (Shanghai, China). The siRNA sequences were as follows: siRNA-1, 5'-GCTAGACACATCCAAGAAAGG-3'; siRNA-2, 5'-GCCGAAAGCATCAAGTCAAAG-3'; siRNA-3, 5'-GCC GACCTATGTCAAGGTTGA-3'; siRNA-4, 5'-GGAAGTTGG CCATCGAGAACA-3'; and scrambled siRNA, 5'-TTCTCC GAACGTGTCACGT-3'. Cells were transfected with siRNAs using Lipofectamine ${ }^{\circledR} 2000$ (Invitrogen; Thermo Fisher Scientific, Inc.), according to the manufacturer's protocol. Following knockdown efficacy evaluation, the 3rd and 4th siRNA variants at $72 \mathrm{~h}$ were selected for subsequent experiments. The groups were named as blank control (BC; untransfected), negative control (NC; scrambled siRNA), SALL4/siRNA-1 and SALL4/siRNA-2. Details of cell culture, construction and transfection siRNA, and evaluation of the siRNAs intervention efficacy were performed as previously described (15).

Protein extraction andwestern blotting. U251 cells were washed with $1 \mathrm{X}$ PBS and pelleted at $12,000 \mathrm{x}$ for $10 \mathrm{~min}$ at $4^{\circ} \mathrm{C}$. Total protein concentration was measured using a Bicinchoninic Assay Protein Assay kit (Beyotime Institute of Biotechnology, Haimen, China). Proteins extracted from cells ( $40 \mu \mathrm{g}$ per lane) were separated using 10\% SDS-PAGE gel (Beyotime Institute of Biotechnology) and transferred to a polyvinylidene fluoride membrane (EMD Millipore, Billerica, MA, USA) at $100 \mathrm{~V}$ for $1 \mathrm{~h}$. Following blocking with 5\% non-fat milk in PBS with $0.1 \%$ Tween (PBST) for $1 \mathrm{~h}$ at room temperature, the membranes were incubated at $4^{\circ} \mathrm{C}$ overnight with primary antibodies and washed three times with 1X TBST. Next, cells were incubated at room temperature for $2 \mathrm{~h}$ with the secondary antibody. The primary antibodies used were as follows: Mouse polyclonal anti-SALL4 (Abgent, Inc., San Diego, CA, USA; cat no. AP1488b, dilution, 1:500); mouse polyclonal anti-OCT4 (cat no. 11263-1-AP), mouse polyclonal anti-NANOG (cat no. 14295-1-AP), mouse polyclonal anti-SOX2 (cat no. 20118-1-AP), mouse polyclonal anti-ABCG2 (cat no. 10051-1-AP) and mouse polyclonal anti-MGMT (cat no. 17195-1-AP) (all from ProteinTech Group, Inc., Chicago, IL, USA; dilution, 1:500); and polyclonal rabbit anti- $\beta$-actin (Santa Cruz Biotechnology, Inc., Dallas, TX , USA; cat no. sc-130656, 1:1,000) used as a gel loading control. The secondary antibody used was horseradish peroxidise-conjugated Goat Anti-Rabbit IgG (H\&L) AP (BioVision, Inc., San Francisco, CA, USA; cat no. 93-6923-100, dilution, 1:2,000). Immunoblotting bands were visualized visualized using ECL Substrates (Tanon Science and Technology Co., Ltd, Shanghai, China) and quantified using ImageJ software version 1.48 (National Institutes of Health, Bethesda, MA, USA).

Reverse transcription-quantitative polymerase chain reaction $(R T-q P C R)$ analysis. Total RNA was extracted from U251 glioma cells using TRIzol ${ }^{\circledR}$ reagent (Invitrogen; Thermo Fisher Scientific, Inc., Waltham, MA, USA). Reverse transcription was performed using the Thermoscript RT-PCR system (Invitrogen; Thermo Fisher Scientific, Inc.) with random hexamer primers and the Superscript II Reverse Transcriptase kit (Invitrogen; Thermo Fisher Scientific, Inc.), for $30 \mathrm{~min}$ at $25^{\circ} \mathrm{C}$, $30 \mathrm{~min}$ at $42^{\circ} \mathrm{C}$ and $10 \mathrm{~min}$ at $85^{\circ} \mathrm{C}$. qPCR was performed using the quantitative Real Time PCR 7500 sequence detection system (Applied Biosystems, Foster city, CA, USA). RT-qPCR was performed as follows: 40 cycles of denaturation at $95^{\circ} \mathrm{C}$ $(12 \mathrm{sec})$ and annealing/extension at $60^{\circ} \mathrm{C}(40 \mathrm{sec})$. Primers were designed using Primer 5.0 software (Applied Biosystems). The primer sequences were as follows: SALL4 forward (F), 5'-ATAGTCAAGCCGAAAGCATCA AGTC-3' and reverse (R), 5'-CTCCGACCTTCCATCTCAGTGC-3'; OCT4 F, 5'-ACCTATTCAGCCAAACGACCAT-3' and R, 5'-CTG CTTCCTCCACCCACTTCT-3'; SOX2 F, 5'-GCTCGCAGA CCTACATGAAC-3' and R, 5'-GGGAGGAAGAGGTAACCA CA-3'; NANOGF, 5'-ATACCTCAGCCTCCAGCAGATG-3' and R, 5'-TTCTGCCACCTCTTAGATTTCATTC-3'; MGMT F, 5'-TCTTCACCATCCCGTTTTCCAG-3' and R, 5'-CTT CTCCGAATTTCACAACCTTCAG-3'; ABCG2 F, 5'-GAA ACCTGGTCTCAACGCCATC-3' and R, 5'-ACTTGGATCT TTCCTTGCAGCTAAG-3'; and GAPDH F, 5'-CATGAGAAG TATGACAACAGCCT-3' and R, 5'-AGTCCTTCCACGATA CCAAAGT-3'. SALL4 mRNA expression was normalized to GAPDH and analyzed using the $2^{-\Delta \Delta \mathrm{Cq}}$ method (16).

Cell cycle analysis. Cell cycle analysis was performed by flow cytometry using transfected and control U251 cells in $\log$-phase growth. Cells were washed with PBS, fixed with $90 \%$ ethanol overnight at $4^{\circ} \mathrm{C}$ and incubated with RNase at $37^{\circ} \mathrm{C}$ for $30 \mathrm{~min}$. Cell nuclei were stained with propidium iodide (PI) at $4^{\circ} \mathrm{C}$ for an additional $30 \mathrm{~min}$. The stained nuclei were then analyzed using a FACSCalibur flow cytometer (BD Biosciences, Franklin Lakes, NJ, USA). The populations of cells distributed in the $G_{0} / G_{1}, S$ and $G_{2} / M$ cell cycle phases were evaluated using WinMDI version 2.9 software (The Scripps Research Institute, San Diego, CA, USA).

Apoptosis assay. Apoptosis was evaluated using an annexin V fluorescein isothiocyanate (FITC)/PI apoptosis detection kit (Thermo Fisher Scientific, Inc.), according to the manufacture's protocol. Briefly, following incubation $(16 \mathrm{~h})$ at $37^{\circ} \mathrm{C}$ in a $5 \% \mathrm{CO}_{2}$ humidified atmosphere, U251 cells cultured in 6-cm dishes were digested with trypsin without EDTA, washed twice with PBS and suspended in $100 \mu 1$ binding buffer, followed by staining with $5 \mu \mathrm{l}$ Annexin V-FITC and $5 \mu \mathrm{l}$ PI for $15 \mathrm{~min}$ in the dark at room temperature and then analyzed by flow cytometry as aforementioned.

Invasion assay. Equal numbers $\left(1 \times 10^{5}\right)$ of transfected and control U251 cells were seeded in separate 24-well cell culture inserts coated with Matrigel with $8 \mu \mathrm{m}$ pores. $500 \mu \mathrm{l}$ DMEM supplemented with $10 \%$ FBS was added into the lower chamber as a chemoattractant. Following a $24-\mathrm{h}$ incubation at $37^{\circ} \mathrm{C}$ with $5 \% \mathrm{CO}_{2}$, cells that were adhered to the upper surface of the filter were removed using a cotton applicator. The cells on the lower surface of the membrane (the migrated cells) were fixed with $3.7 \%$ formaldehyde for $10 \mathrm{~min}$ at $-20^{\circ} \mathrm{C}$, stained with $0.1 \%$ crystal violet (Beyotime Institute of Biotechnology) for $30 \mathrm{~min}$ at $37^{\circ} \mathrm{C}$. The cell number was determined in at least five 
randomly selected fields under a light microscope (magnification, x200; SZ61; Olympus, Tokyo, Japan). The invasion rate was determined for three independent experiments as follows: No. of migrated cells/total no. of cells x100.

Cytotoxicity assay. The cytotoxicity of TMZ (Merck \& Co., Inc., Whitehouse Station, NJ, USA) on glioma cells was detected using a Total Superoxide Dismutase Assay kit and WST-8 assay according to manufacturers' protocol. Briefly, U251 cells were plated at a density of $5 \times 10^{3}$ cells/well in 96-well plates and allowed to attach overnight at $37^{\circ} \mathrm{C}$. Various concentrations of TMZ $(2,4,8,16,32,64,128$ and $256 \mu \mathrm{g} / \mathrm{ml})$ were subsequently added, and the cells were cultured for $72 \mathrm{~h}$ at $37^{\circ} \mathrm{C}$. A total of 4 -h prior to harvest, $10 \mu \mathrm{l} /$ well of the Cell Counting kit- 8 reagent (Dojindo Molecular Technologies, Inc., Kumamoto, Japan) was added and the cells were incubated for $2 \mathrm{~h}$ at $37^{\circ} \mathrm{C}$. The optical density (OD) at $450 \mathrm{~nm}$ was recorded using a microplate reader (Bio-Rad). The cell survival rate was determined by comparing the OD values of the treated samples with those of the untreated controls within each group. The concentration of TMZ required to inhibit cell growth by $50 \%$ (the toxic concentration, $\mathrm{TC}_{50}$ ) was evaluated using survival curves.

Statistical analysis. Data are expressed as the mean \pm standard deviation of three independent experiments. Statistical analysis was performed using the Student's t-test between two groups, whereas the comparison between $\geq$ three groups was performed using one-way analysis of variance. Post hoc tests were used for comparisons between groups. Student-Neuman-Keuls method was used when equal variances assumed. If equal variances not assumed, Dunnett's T3 method was used. All analyses were performed using SPSS version 13.0 software (SPSS, Inc., Chicago, IL, USA). P<0.05 was considered to indicate a statistically significant difference.

\section{Results}

SALL4 knockdown induces cell cycle arrest, enhances apoptosis and invasion inhibition. The cell cycle distribution in various transfection groups of U251 glioma cells were analyzed by flow cytometry $72 \mathrm{~h}$ after transfection. No significant differences in the fraction of $\mathrm{G}_{1}$ phase cells were identified between the $\mathrm{NC}$ group $(52.11 \pm 1.92 \%)$ and $\mathrm{BC}$ group $(42.30 \pm 0.42 \%)(\mathrm{P}=0.14$; Fig. 1A). However, following treatment with siRNAs, the $\mathrm{G}_{1}$ phase cell fraction in the SALL4/siRNA-1 $(77.17 \pm 1.77 \%)$ and SALL4/siRNA-2 $(76.67 \pm 1.12 \%)$ groups were significantly higher compared with that of the $\mathrm{BC}$ (both $\mathrm{P}<0.001)$ and $\mathrm{NC}$ groups (siRNA-1, $\mathrm{P}=0.002$; siRNA-2, $\mathrm{P}=0.005$ ) (Fig. 1A). Correspondingly, the fraction of cells in the $S$ phase were $36.75 \pm 0.95,31.03 \pm 3.88$, $10.54 \pm 1.08$ and $9.76 \pm 1.16 \%$ in the BC, NC, SALL4/siRNA-1, and SALL4/siRNA-2 groups, respectively. Additionally, the reduction in the fraction of cells in the $\mathrm{G}_{2}-\mathrm{M}$ phase were $18.41 \pm 2.10,17.17 \pm 2.01,12.28 \pm 2.84$ and $13.58 \pm 1.10 \%$ in the BC, NC, SALL4/siRNA-1, and SALL4/siRNA-2 groups, respectively. SALL4/siRNA-1 and SALL4/siRNA-2 demonstrated significantly lower $\mathrm{S}$ phase cell proportions compared with those in the $\mathrm{BC}$ (both $\mathrm{P}<0.001$ ) and $\mathrm{NC}$ groups
(siRNA-1, P=0.047; siRNA-2, $\mathrm{P}=0.041$ ). However, no significant differences in the $\mathrm{G}_{2}-\mathrm{M}$ cell cycle phase were identified in the SALL4/siRNA-1 and SALL4/siRNA-2 groups, when compared with the BC (siRNA-1, $\mathrm{P}=0.24$; siRNA-2, $\mathrm{P}=0.21$ ), and NC (siRNA-1, $\mathrm{P}=0.39$; siRNA-2, $\mathrm{P}=0.36$ ) groups.

Subsequently, the present study compared the apoptosis rate prior to and following SALL4 knockdown (Fig. 1B). It was revealed that SALL4/siRNA-1 $(85.88 \pm 0.54 \%)$ and SALL4/siRNA-2 $(86.41 \pm 0.87 \%)$ induced significantly increased early apoptosis rates compared with that in the $\mathrm{BC}$, and $\mathrm{NC}$ groups (all $\mathrm{P}<0.001$ ).

The Transwell migration was performed to further examine the effect of SALL4 on glioma cell invasion (Fig. 1C). The mean invasion cell number was $94.33 \pm 3.51$ and $91.33 \pm 1.53$ in the $\mathrm{BC}$, and $\mathrm{NC}$ groups, respectively, compared with $45.00 \pm 1.00$ and 47.67 3.06 in the SALL4/siRNA-1, and SALL4/siRNA-2 groups, respectively. The siRNA treatment groups significantly reduced the migratory ability of cells compared with that in BC (siRNA-1, P=0.005; siRNA-2, P<0.001) and NC (siRNA-1, $\mathrm{P}=0.001$; siRNA-2, $\mathrm{P}<0.001)$ groups.

The Janus kinase (Jak)-signal transducer and activator of transcription 3 (Stat3) signaling pathway is utilized by SALL4 to fulfill its function. In order to investigate the reduction of malignancy of glioma detected in the present study, the expression levels of OCT4, SOX2 and NANOG were analyzed, which are essential factors of the Jak-Stat3 signaling pathway. It was demonstrated that SALL4/siRNA-1 and SALL4/siRNA-2 suppressed the expression levels of OCT4, SOX2 and NANOG, which were all statistically lower compared with the $\mathrm{BC}$ and $\mathrm{NC}$ groups (Fig. 2A). Compared with the BC group, the inhibition rates of SALL4/siRNA-1 on OCT4, SOX2 and NANOG mRNA expression levels were 59\% $(\mathrm{P}=0.004)$, $38 \%(\mathrm{P}=0.001)$ and $39 \%(\mathrm{P}=0.002)$, respectively, whereas the inhibition rates of SALL4/siRNA-2 were $65 \%(\mathrm{P}<0.001), 26 \%$ $(\mathrm{P}=0.038)$ and $29 \%(\mathrm{P}=0.036)$, respectively. Compared with the NC group, the inhibition rates of SALL4/siRNA-1 were 65.54\% $(\mathrm{P}<0.001), 37.37 \%(\mathrm{P}=0.038)$ and $40.20 \%(\mathrm{P}=0.013)$, respectively, whereas the inhibition rates of SALL4/siRNA-2 were $70.59 \%(\mathrm{P}<0.001), 25.25 \%(\mathrm{P}=0.037)$ and $30.40 \%$ $(\mathrm{P}=0.032)$ (Table I; Fig. 2A). Western blot analysis revealed similar results (Fig. 2B).

SALL4 knockdown increases chemosensitivity to TMZ. Subsequently, the present study aimed to investigate the effect of SALL4 on chemotherapeutic TMZ during glioma treatment. It was revealed that the $\mathrm{TC}_{50}$ of $\mathrm{TMZ}$ in SALL4/siRNA-1 and SALL4/siRNA-2 groups were $68.34 \pm 3.52$, and $67.44 \pm 4.71 \mu \mathrm{g} / \mathrm{ml}$, respectively. Compared with that in the $\mathrm{BC}(113.66 \pm 23.07 \mu \mathrm{g} / \mathrm{ml})$ and $\mathrm{NC}$ groups $(114.93 \pm 20.91 \mu \mathrm{g} / \mathrm{ml})$, the $\mathrm{TC}_{50}$ value of SALL4/siRNA-1 (BC, $\mathrm{P}=0.047$; NC, $\mathrm{P}=0.030$ ) and SALL4/siRNA-2 were significantly decreased $(\mathrm{BC}, \mathrm{P}=0.043$; $\mathrm{NC}, \mathrm{P}=0.026$ ) (data not shown).

In order to describe this phenomenon, the present study hypothesized that the knockdown of SALL4 suppressed glioma tumor resistance via MGMT and ABCG2 downregulation. The present study revealed that SALL4/siRNA-1 decreased MGMT and ABCG2 mRNA expression levels by 39 , and $28 \%$, respectively, which were significantly lower 

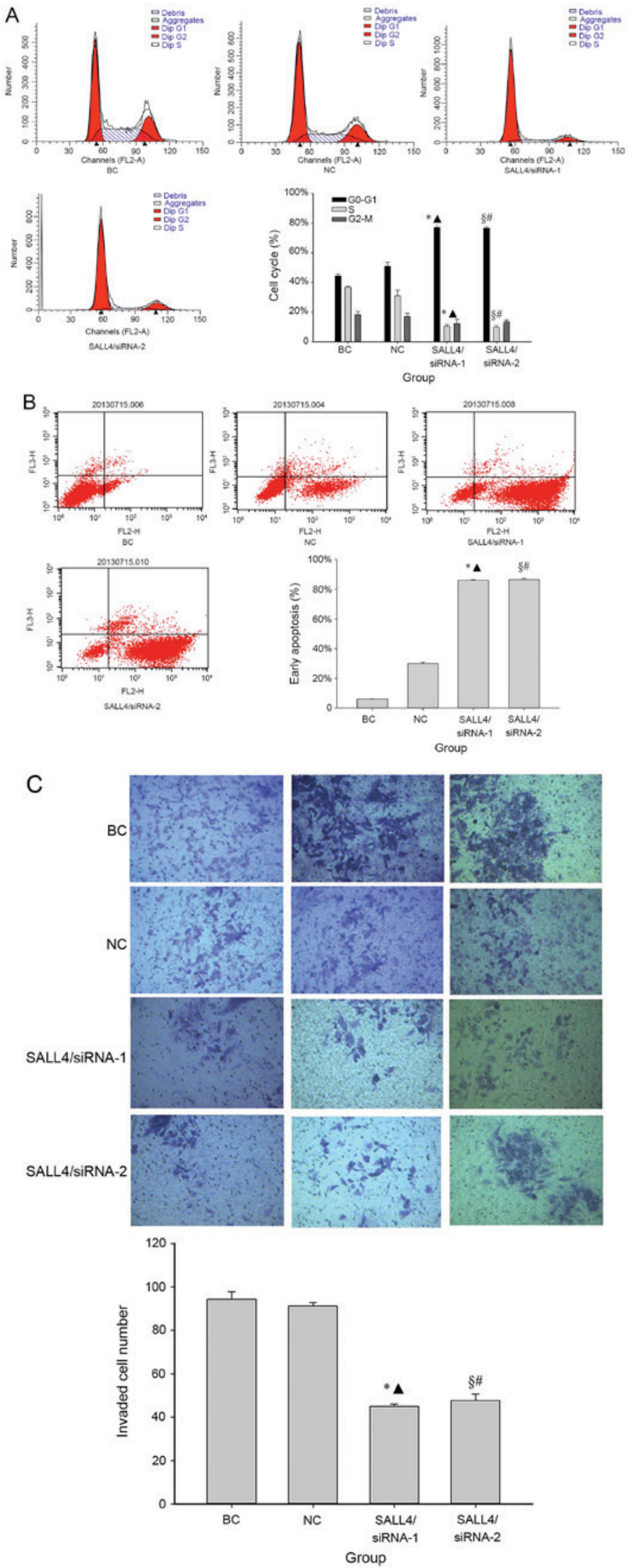

Figure 1. SALL4 knockdown induced cell cycle arrest, enhanced early apoptosis and inhibited invasion. (A) Inhibition effects of SALL4 on cell cycle distribution. The cells were analyzed by flow cytometry at $72 \mathrm{~h}$ after transfection. Histograms reveal G0/G1 cell cycle phase arrest and a decline in S phase in siRNA groups. (B) Knockdown of SALL4 increased the early apoptosis rate in U251 cells. Apoptosis rate was evaluated in fluorescein isothiocyanate-annexin V and propidium-stained cells by flow cytometry. Apoptotic cell numbers were significantly higher in siRNA groups. (C) Knockdown of SALL4 expression level decreased cell invasive capacity of U251 cells. Matrigel assays demonstrate the effect on the invasive potential in each group. The histogram indicated the mean number of invaded cells at $72 \mathrm{~h}$ after top-side cell seeding, which revealed that the capacity of invasion was significantly suppressed in both siRNA groups. BC: untransfected; NC: scrambled siRNA. ${ }^{*} \mathrm{P}<0.05$ siRNA-1 vs. $\mathrm{BC},{ }^{\mathbf{A}} \mathrm{P}<0.05$ siRNA-1 vs. NC, ${ }^{{ }^{P}} \mathrm{P}<0.05$ siRNA-2 vs. BC, ${ }^{\text {"P }}<0.05$ siRNA-2 vs. NC. SALL4, spalt-like transcription factor 4; siRNA, short interfering RNA; BC, blank control; NC, negative control. 

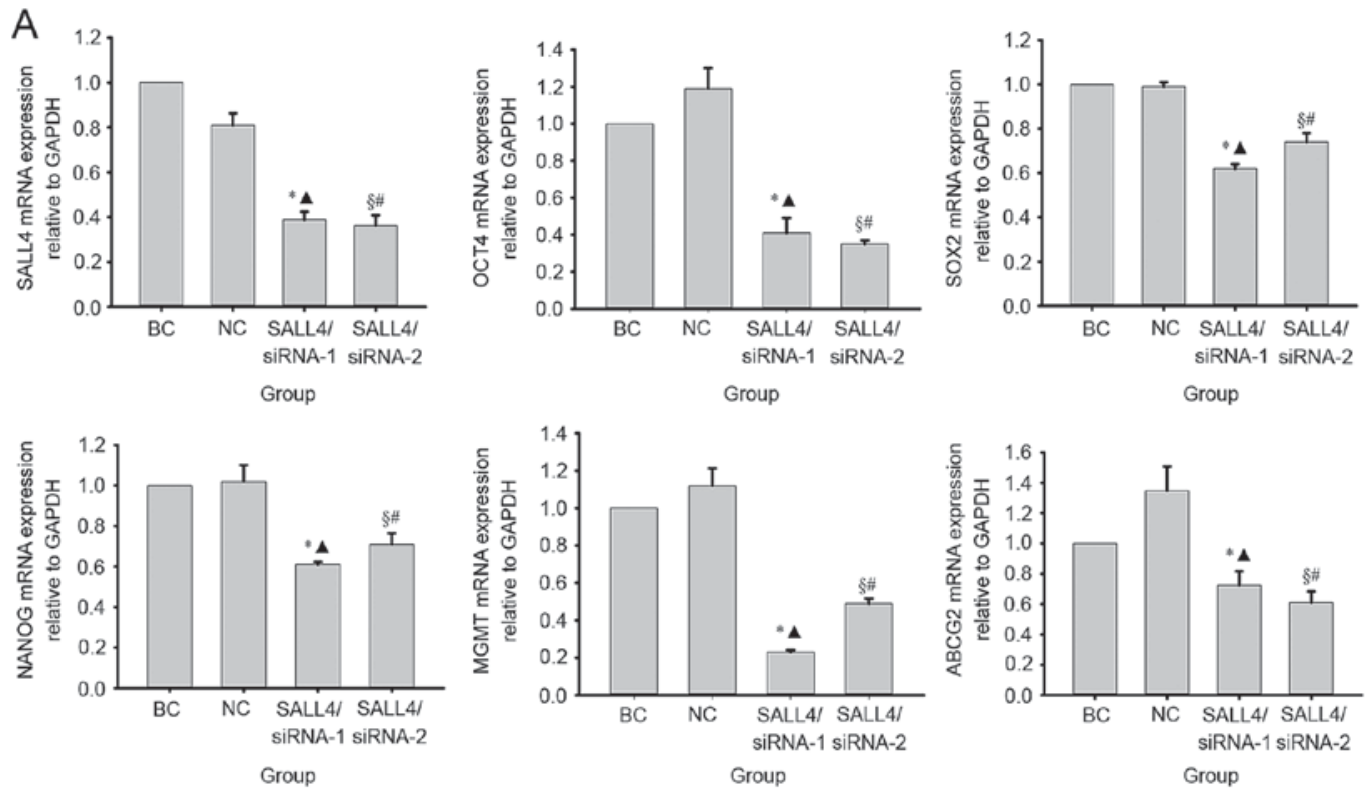

B
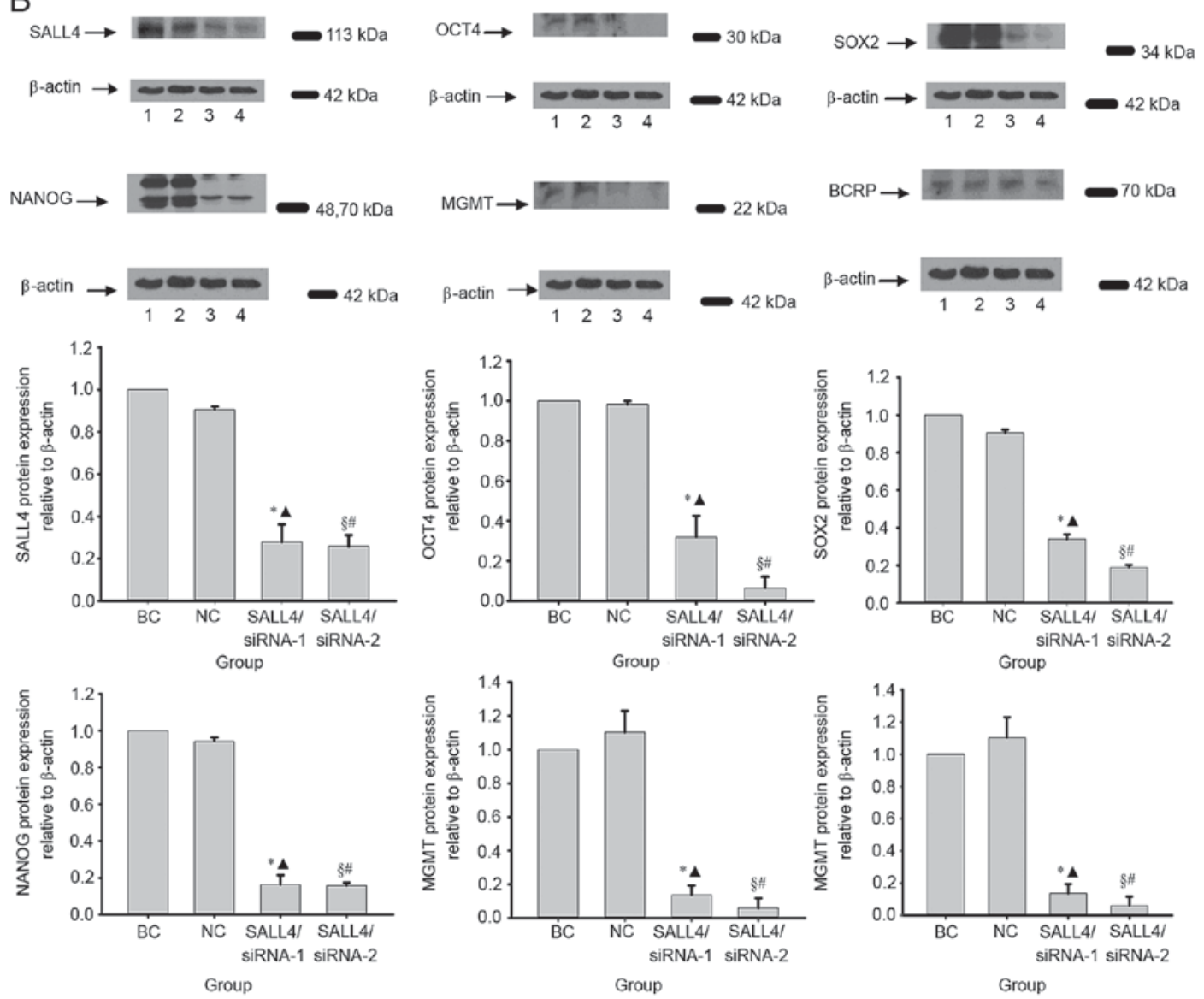

Figure 2. SALL4 knockdown altered the expression levels of OCT4, SOX2, NANOG, MGMT and ABCG2. (A) The mRNA expression levels of OCT4, SOX2, NANOG, MGMT and ABCG2 in siRNA groups was markedly lower compared with in BC and NC groups at $72 \mathrm{~h}$ post-transfection. (B) Western blotting revealed that changes in expression levels at the protein level were qualitatively similar to the changes in mRNA expression levels. BC group; NC group; SALL4/siRNA-1 group; SALL4/siRNA-2 group. ${ }^{*} \mathrm{P}<0.05$ siRNA-1 vs. BC, ${ }^{\mathbf{A}} \mathrm{P}<0.05$ siRNA-1 vs. NC, ${ }^{\circ} \mathrm{P}<0.05$ siRNA-2 vs. BC, ${ }^{\prime \prime} \mathrm{P}<0.05$ siRNA-2 vs. NC. SALL4, spalt-like transcription factor 4; OCT4, POU class 5 homeobox 1; SOX2, SRY-box 2; NANOG, Nanog homeobox; MGMT, O-6-methylguanine-DNA methyltransferase; ABCG2, adenosine triphosphate-binding cassette subfamily G member 2; siRNA, short interfering RNA; BC, blank control; NC, negative control.

compared with that of the $\mathrm{BC}$ (MGMT, $\mathrm{P}=0.021 ; \mathrm{ABCG}$, $\mathrm{P}=0.032)$ and $\mathrm{NC}(\mathrm{MGMT}, \mathrm{P}<0.001 ; \mathrm{ABCG} 2, \mathrm{P}=0.024)$ groups (Table I; Fig. 2A). Similarly, when compared with the BC and NC groups, SALL4/siRNA-1 decreased MGMT protein expression level significantly (BC, $\mathrm{P}=0.009 ; \mathrm{NC}, \mathrm{P}=0.010)$ and SALL4/siRNA-2 significantly decreased expression levels of MGMT (BC, $\mathrm{P}=0.007$; $\mathrm{BC}, \mathrm{P}=0.009)$ and $\mathrm{ABCG} 2$ (BC, $\mathrm{P}=0.009 ; \mathrm{BC}, \mathrm{P}=0.006$ ) (Fig. 2B). 
Table I. mRNA expression levels relative to GAPDH.

Gene

\begin{tabular}{lcccccc}
\cline { 2 - 6 } Group & SALL4 & OCT4 & SOX2 & NANOG & MGMT & ABCG2 \\
\hline BC & 1 & 1 & 1 & 1 & 1 & 1 \\
NC & $0.81 \pm 0.05$ & $1.19 \pm 0.11$ & $0.99 \pm 0.02$ & $1.02 \pm 0.08$ & $1.02 \pm 0.08$ & $1.35 \pm 0.16$ \\
SALL4/siRNA-1 & $0.39 \pm 0.03$ & $0.41 \pm 0.08$ & $0.62 \pm 0.02$ & $0.61 \pm 0.01$ & $0.61 \pm 0.01$ & $0.72 \pm 0.09$ \\
SALL4/siRNA-2 & $0.36 \pm 0.05$ & $0.35 \pm 0.02$ & $0.74 \pm 0.04$ & $0.71 \pm 0.05$ & $0.71 \pm 0.05$ & $0.61 \pm 0.07$ \\
\hline
\end{tabular}

SALL4, spalt-like transcription factor 4; OCT4, POU class 5 homeobox 1; SOX2, SRY-box 2; NANOG, Nanog homeobox; MGMT, O-6-methylguanine-DNA methyltransferase; ABCG2, adenosine triphosphate-binding cassette subfamily G member 2; BC, blank control; NC, negative control.

\section{Discussion}

Since SALL4 was first detected as an oncogene in leukemia (17), the function of SALL4 had been studied in various types of cancer. For example, in lung, breast, gastric and colorectal cancer cells (10-12), SALL4 has been demonstrated to regulate cell viability, apoptosis, and tumorigenicity (12). In the authors' previous study, it was reported that SALL4 served an essential role in glioma and its expression was negatively associated with prognosis (15); however, the detailed mechanisms remain unclear. The present study aimed to investigate the detailed mechanisms underlying SALL4 in glioma tumorigenesis. Following knockdown of SALL4, cell cycle arrest, significantly increased levels of early apoptosis and invasion inhibition were observed in GBM cells. Furthermore, decreased SALL4 expression level significantly decreased mRNA and protein expression levels of OCT4, SOX2, and NANOG. In addition, inhibition of SALL4 decreased the $\mathrm{TC}_{50}$ of chemotherapeutic agent TMZ. These results partially elucidated the specific mechanisms utilized by SALL4, indicating that SALL4 may serve an important role in GBM tumorigenesis.

The CSC theory hypothesizes that CSCs and ESCs share numerous malfunctioned proliferation signal pathways (18), and the Jak-Stat 3 signaling pathway is considered as one of the most important (19). At the top hierarchy of the Jak-Stat3 signaling pathway, OCT4, SOX2 and NANOG, are considered to serve the most important function in maintaining ESC properties (20). Previously, increased glioma progression was identified to be associated with upregulated OCT4, SOX2 and NANOG $(21,22)$. Additionally, combinatorial expression levels of OCT4, NANOG and SOX2 were positively associated with increasing glioma malignancy (22). The present study demonstrated that knockdown of SALL4 significantly downregulated the mRNA and protein expression levels of OCT4, SOX2, and NANOG, which supports the results regarding SALL4 involvement in cell cycle arrest, enhanced apoptosis and invasion inhibition. These data suggest that SALL4 inhibits glioma tumorigenesis by participating in the Jak-Stat3 signaling pathway. However, it remains unclear how and which factors SALL4 interacts with in the Jak-Stat3 signaling pathway; therefore, further research is required for elucidation.
Notably in the present study, the knockdown of SALL4 significantly reduced the $\mathrm{TMZ} \mathrm{TC}_{50}$. As previously demonstrated, cancer utilizes various mechanisms enabling resistance to anticancer drugs, including DNA repairing and reducing toxin uptake via pumping-out chemotherapeutics (23). TMZ is a classical chemotherapy drug for glioma, which is normally used by patients with GBM followed by surgical resection and radiotherapy (24). However, the majority of patients with glioma develop resistance to TMZ during treatment (25). Thus, it was hypothesized that glioma may use or develop 'DNA repairing' and 'toxin pumping-out' strategies to remain resistant. MGMT is a DNA repair protein, which removes alkylating adducts from the O6 position of guanine and protects cells from cytotoxic, and mutagenic effects, resulting in a resistance of tumor cells to alkylating agent-based chemotherapy (26). Following the knockdown of SALL4, a significant decrease in the $\mathrm{TC}_{50}$ of $\mathrm{TMZ}$ with simultaneous inhibition of MGMT expression was observed. A previous study demonstrated that the expression level of SALL4 was positively associated with histone deacetylase activity in EpCAM-positive hepatocellular carcinomacell Hep3B and $\mathrm{HuH} 7$ cell lines (27). As the present study knocked down SALL4 in glioma cells, there would be less potent histone deacetylase activity, which may inhibit various protein expression levels. Although it remained unclear whether MGMT promoter would be deacetylated, the present study observed a lowered $\mathrm{TC}_{50}$ for $\mathrm{TMZ}$ as well as decreased MGMT protein expression levels simultaneously. Thus, it was reasonable to hypothesize that the knockdown of SALL4 decreased histone deacetylase activity, which inhibited MGMT expression and the subsequent $\mathrm{TMZ} \mathrm{TC}_{50}$ in glioma cells. This hypothesis is supported by the results of a previous study that also used glioma cells (28) and the results of the present study. Additionally, pumping out chemotherapeutics is another popular mechanism used by various types of cancer. ABC drug transporters have been accepted to reduce the efficacy of chemotherapeutics by pumping out toxic drugs and thus contribute to aggressive tumor behaviors, and poor prognosis (29). As previously demonstrated to be enriched in GBM (30), ABCG2, which is a member of the ABC family, could also be involved in tumor resistance. The present study demonstrated that following knockdown of SALL4, ABCG2 exhibited significantly reduced expression levels, which may 
explain the reduction of $\mathrm{TMZ} \mathrm{TC}_{50}$. Thus, the results of the present study suggest that MGMT and ABCG2 serve important roles in GBM resistance, and SALL4 is responsible for maintaining high expression and activity levels of MGMT and ABCG2. Further studies should focus on where and how SALL4 interacts with MGMT and ABCG2.

In conclusion, the present study revealed that the knockdown of SALL4 significantly decreased the malignancy and increased the sensitivity of TMZ to U251 cells. These results indicate that SALL4 may serve an essential role in the tumorigenic properties of glioma cells. Thus, SALL4 may be a potential therapeutic target for glioma, particularly for GBM.

\section{Acknowledgements}

The present study was supported by the National Natural Science Foundation of China (grant no. 30930094) and the Natural Science Foundation of Shanghai (grant no. 13ZR1414200).

\section{References}

1. Weller M: Novel diagnostic and therapeutic approaches to malignant glioma. Swiss Med Wkly 141: w13210, 2011.

2. Louis DN, Ohgaki H, Wiestler OD, Cavenee WK, Burger PC, Jouvet A, Scheithauer BW and Kleihues P: The 2007 WHO classification of tumours of the central nervous system. Acta Neuropathol 114: 97-109, 2007.

3. Wen P and Kesari S: Malignant gliomas in adults. N Engl J Med 359: 492-507, 2008.

4. Kohlhase J, Schuh R, Dowe G, Kühnlein RP, Jäckle H, Schroeder B, Schulz-Schaeffer W, Kretzschmar HA, Köhler A Müller U, et al: Isolation, characterization, and organ-specific expression of two novel human zinc finger genes related to the Drosophila gene spalt. Genomics 38: 291-298, 1996.

5. Zhang J, Tam WL, Tong GQ, Wu Q, Chan HY, Soh BS, Lou Y, Yang J, Ma Y, Chai L, et al: Sall4 modulates embryonic stem cell pluripotency and early embryonic development by the transcriptional regulation of Pou5f1. Nat Cell Biol 8: 1114-1123, 2006.

6. Yang J, Gao C, Chai L and Ma Y: A novel SALL4/OCT4 transcriptional feedback network for pluripotency of embryonic stem cells. PLoS One 5: e10766, 2010.

7. Singh SK, Clarke ID, Terasaki M, Bonn VE, Hawkins C, Squire J and Dirks PB: Identification of a cancer stem cell in human brain tumors. Cancer Res 63: 5821-5828, 2003.

8. Galli R, Binda E, Orfanelli U, Cipelletti B, Gritti A, De Vitis S, Fiocco R, Foroni C, Dimeco F and Vescovi A: Isolation and characterization of tumorigenic, stem-like neural precursors from human glioblastoma. Cancer Res 64: 7011-7021, 2004.

9. Al-Hajj M, Wicha MS, Benito-Hernandez A, Morrison SJ and Clarke MF: Prospective identification of tumorigenic breast cancer cells. Proc Natl Acad Sci USA 100: 3983-3988, 2003.

10. Kobayashi D, Kuribayashi K, Tanaka M and Watanabe N: Overexpression of SALL4 in lung cancer and its importance in cell proliferation. Oncol Rep 26: 965-970, 2011.

11. Kobayashi D, Kuribayshi K, Tanaka M and Watanabe N: SALL4 is essential for cancer cell proliferation and is overexpressed at early clinical stages in breast cancer. Int J Oncol 38: 933-939, 2011.

12. Yong KJ, Gao C, Lim JS, Yan B, Yang H, Dimitrov T, Kawasaki A, Ong CW, Wong KF, Lee S, et al: Oncofetal gene SALL4 in aggressive hepatocellular carcinoma. N Engl J Med 368 2266-2276, 2013.
13. Aguila JR, Liao W, Yang J, Avila C, Hagag N, Senzel L and Ma Y: SALL4 is a robust stimulator for the expansion of hematopoietic stem cells. Blood 118: 576-585, 2011.

14. Yang J, Aguila JR, Alipio Z,Lai R, Fink LM and Ma Y: Enhanced self-renewal of hematopoietic stem/progenitor cells mediated by the stem cell gene Sall4. J Hematol Oncol 4: 38, 2011.

15. Zhang L, Yan Y, Jiang Y, Cui Y, Zou Y, Qian J, Luo C, Lu Y and $\mathrm{Wu} \mathrm{X}$ : The expression of SALL4 in patients with gliomas: High level of SALL4 expression is correlated with poor outcome. J Neurooncol 121: 261-268, 2015.

16. Livak KJ and Schmittgen TD: Analysis of relative gene expression data using real-time quantitative PCR and the 2(-Delta Delta C(T)) method. Methods 25: 402-408, 2001.

17. Ma Y, Cui W, Yang J, Qu J, Di C, Amin HM, Lai R, Ritz J, Krause DS and Chai L: SALL4, a novel oncogene, is constitutively expressed in human acute myeloid leukemia (AML) and induces AML in transgenic mice. Blood 108: 2726-2735, 2006.

18. Cheng L, Alexander R, Zhang S, Pan CX, MacLennan GT, Lopez-Beltran A and Montironi R: The clinical and therapeutic implications of cancer stem cell biology. Expert Rev Anticancer Ther 11: 1131-1143, 2011.

19. Yu H, Lee H, Herrmann A, Buettner R and Jove R: Revisiting STAT3 signalling in cancer: New and unexpected biological functions. Nat Rev Cancer 14: 736-746, 2014

20. Boyer LA, Lee TI, Cole MF, Johnstone SE, Levine SS, Zucker JP, Guenther MG, Kumar RM, Murray HL, Jenner RG, et al: Core transcriptional regulatory circuitry in human embryonic stem cells. Cell 122: 947-956, 2005.

21. Guo Y, Liu S, Wang P, Zhao S, Wang F, Bing L, Zhang Y, Ling EA, Gao J and Hao A: Expression of embryonic stem cell-associated genes Oct4, Sox 2 and Nanog in human gliomas. Histopathology 59: 763-775, 2011.

22. Holmberg J, He X, Peredo I, Orrego A, Hesselager G, Ericsson C, Hovatta O, Oba-Shinjo SM, Marie SK, Nistér M and Muhr J: Activation of neural and pluripotent stem cell signatures correlates with increased malignancy in human glioma. PLoS One 6: e18454, 2011.

23. Shen DW, Pouliot LM, Hall MD and Gottesman MM: Cisplatin resistance: A cellular self-defense mechanism resulting from multiple epigenetic and genetic changes. Pharmacol Rev 64: 706-721, 2012.

24. Stupp R, Mason WP, van den Bent MJ, Weller M, Fisher B, Taphoorn MJ, Belanger K, Brandes AA, Marosi C, Bogdahn U, et al: Radiotherapy plus concomitant and adjuvant temozolomide for glioblastoma. N Engl J Med 352: 987-996, 2005.

25. Hiddingh L, Raktoe RS, Jeuken J, Hulleman E, Noske DP, Kaspers GJ, Vandertop WP, Wesseling P and Wurdinger T: Identification of temozolomide resistance factors in glioblastoma via integrative miRNA/mRNA regulatory network analysis. Sci Rep 4: 5260, 2014.

26. Kaina B, Christmann M, Naumann S and Roos WP: MGMT: Key node in the battle against genotoxicity, carcinogenicity and apoptosis induced by alkylating agents. DNA Repair (Amst) 6: 1079-1099, 2007

27. Zeng SS, Yamashita T, Kondo M, Nio K, Hayashi T, Hara Y, Nomura Y, Yoshida M, Hayashi T, Oishi N, et al: The transcription factor SALL4 regulates stemness of EpCAM-positive hepatocellular carcinoma. J Hepatol 60: 127-134, 2014.

28. Kitange GJ, Mladek AC, Carlson BL, Schroeder MA, Pokorny JL, Cen L, Decker PA, Wu W, Lomberk GA, Gupta SK, et al: Inhibition of histone deacetylation potentiates the evolution of acquired temozolomide resistance linked to MGMT upregulation in glioblastoma xenografts. Clin Cancer Res 18: 4070-4079, 2012.

29. Kim HS, Kim NC, Chae KH, Kim G, Park WS, Park YK and Kim YW: Expression of multidrug resistance-associated protein 2 in human gallbladder carcinoma. Biomed Res Int 2013: 527534, 2013.

30. Chamberlain MC, Bota DA, Linskey ME and Schwartz PH: Neural stem/progenitors and glioma stem-like cells have differential sensitivity to chemotherapy. Neurology 77: e135-e136, 2011. 